\title{
Pharmacogenetic role of $X R C C 1$ polymorphisms on the clinical outcome of gastric cancer patients with platinum-based chemotherapy: a systematic review and meta-analysis
}

\author{
J. Xu' ${ }^{1}$, J. Ma ${ }^{2}$, H.T. Zong ${ }^{2}$, S.Y. Wang ${ }^{1}$ and J.W. Zhou ${ }^{1}$ \\ ${ }^{1}$ Department of Molecular Cell Biology and Toxicology, \\ Jiangsu Key Lab of Cancer Biomarkers, Prevention \& Treatment, Cancer Center, \\ School of Public Health, Nanjing Medical University, Nanjing, China \\ ${ }^{2}$ The First Affiliated Hospital of Nanjing Medical University, Nanjing, China \\ Corresponding author: S.Y. Wang \\ E-mail: sywang@njmu.edu.cn
}

Genet. Mol. Res. 13 (1): 1438-1446 (2014)

Received January 4, 2013

Accepted September 20, 2013

Published March 6, 2014

DOI http://dx.doi.org/10.4238/2014.March.6.2

\begin{abstract}
It is still controversial whether X-ray repair crosscomplementing group (XRCC1) gene polymorphisms (Arg194Trp and Arg399Gln) are associated with the clinical outcome of platinumbased chemotherapy in gastric cancer patients based on published studies. Meta-analysis was performed to provide a systematic review of the findings. Eligible articles from the PubMed, SinoMed, and CNKI databases before September 1, 2012, were selected. Objective response (complete response + partial response $v s$ progressive disease + stable disease), progress-free survival (PFS) and overall survival (OS) were applied to evaluate clinical outcomes. We calculated the odds ratio or hazard risk (HR) with $95 \%$ confidence interval (CI) using the STATA software. Eleven eligible articles including 1274 gastric cancer patients with platinum-based treatment were enrolled in our meta-analysis. The results indicated that the A allele of the $\mathrm{XRCCl}$
\end{abstract}


Arg399Gln polymorphism was significantly associated with poor OS $(\mathrm{HR}=1.40 ; 95 \% \mathrm{CI}=1.04-1.90)$ of gastric cancer but not for platinumbased chemotherapy response or PFS. No significant associations were observed between XRCC1 Arg194Trp and objective response. The data suggest that the XRCC1 Arg399Gln polymorphism may be a prognostic biomarker of OS for platinum-based gastric cancer treatment. However, further cohorts with larger sample sizes from different ethnic backgrounds and with improved experimental design are needed to confirm these findings.

Key words: Chemotherapy; $X R C C 1$; Polymorphism; Meta-analysis; Gastric cancer

\section{INTRODUCTION}

Gastric cancer is the fourth most common cancer worldwide and remains the leading cause of cancer death (Parkin et al., 2005). Although radical surgery combined with platinumbased adjuvant chemotherapy has improved clinical outcomes (Sakuramoto et al., 2007), the approximate 5-year survival rate is merely 20\% (Paoletti et al., 2010), and the therapeutic effect differs among individuals.

The DNA repair system plays a critical role in tumorigenesis and treatment resistance (Deans and West, 2011; Wilting and Dannenberg, 2012). XRCC1, the key protein of the base excision repair system, serves as a scaffold protein for repairing base lesions induced by reactive oxygen species (David et al., 2007). We previously identified that gastric cancer patients with higher expression of $X R C C 1$ have a longer survival time than those with low expression (Wang et al., 2012). In addition, XRCC1 and several other DNA repair proteins were involved in repairing cisplatin-induced DNA interstrand cross-links (Zhu and Lippard, 2009). It is conceivable that interindividual differences in XRCC1 may contribute to the capacity of repairing platinum-induced DNA damage, thus influencing the chemotherapy efficacy and clinical outcome of gastric cancer patients.

The most common polymorphisms in the XRCC1 gene are Arg399Gln (rs25487, $\mathrm{G}>\mathrm{A}$ ) and Arg194Trp (rs1799782, C>T). A recent meta-analysis showed that the 2 single nucleotide polymorphisms (SNPs) had predictive value on the clinical outcomes of platinumbased chemotherapy in advanced non-small cell lung cancer (Wu et al., 2012). However, the association between $X R C C 1$ SNPs and clinical outcomes in gastric cancer is contradictory. We conducted a meta-analysis to assess the efficacy of platinum-based chemotherapy of gastric cancer patients with different XRCC1 SNPs.

\section{MATERIAL AND METHODS}

\section{Study selection}

We searched the relevant electronic literature from the PubMed, SinoMed, and CNKI databases before September 1, 2012, using the following searching items: "XRCC1 or X-ray repair cross complementing protein 1", "stomach cancer or gastric cancer", and "polymorphism or variant". 
The eligible studies should meet the following criteria:

a) Advanced, recurrent, or metastatic gastric cancer patients

b) Histology or pathology confirmed

c) Treated with any kinds of platinum drugs

d) Prospective cohort studies of Arg399Gln (G>A) or Arg399Gln polymorphisms

e) Sample size N $>45$.

We also contacted the corresponding authors for missing information and excluded articles lacking critical information after repeated requests (Ott et al., 2011). If the same research group published multiple papers for the same cohort, we selected the paper containing the largest sample size, using the latest polymorphism data, or presenting the most detailed data that could be extracted more easily.

\section{Data extraction and quality assessment}

Two investigators extracted data independently. In some cases with discrepant results, a discussion was initiated to agree on a common conclusion. Available data extracted from each study included the first author's surname, year of publication, country of origin, ethnicity, sample size, genotyping method, and clinical outcome. In addition, ethnicity was categorized as Asian or Caucasian.

\section{Statistical analysis}

We used the World Health Organization (WHO) criteria (Miller et al., 1981) or Response Evaluation Criteria in Solid Tumors (RECIST; Therasse et al., 2000) to estimate the odds ratio (OR) and corresponding $95 \%$ confidence intervals (95\% CIs) for objective response $v s$ no response after platinum-based chemotherapy [complete response + partial response $v s$ progress disease + stable disease]. Pooled Cox proportional hazards ratios (HRs) and 95\%CIs were estimated for progression-free survival (PFS) and overall survival (OS), using published methods (Parmar et al., 1998; Tierney et al., 2007). A $\chi^{2}$ test-based Q-statistic test was performed to check heterogeneity between studies, and $\mathrm{P}<0.10$ was considered to be significant (Lau et al., 1997). Initial analyses with a fixed-effect model were used, and if heterogeneity among studies existed, a random-effect model was applied. Funnel plots and the Egger test were utilized to examine the effect of publication bias. All analyses were performed using the STATA software, and P values were two-side with a significance level of 0.05.

\section{RESULTS}

We initially identified 80 relevant articles, among which only 18 studies met our inclusion criteria through reading the title or abstract. By the detailed assessment, we excluded 1 study for missing data, 1 study due to a small sample size, 1 study for using a regiment without platinum, and 4 studies for duplicate publication (Figure 1).

Only 2 studies, from Italy and Germany, investigated the association between XRCC1 polymorphisms and gastric cancer clinical outcome in the Caucasian population. The patients from the remaining cohorts were Asian, either from China or Korea. In total, 1274 gastric cancer patients had been treated with platinum-based chemotherapy and were included in our analysis (Table 1). 


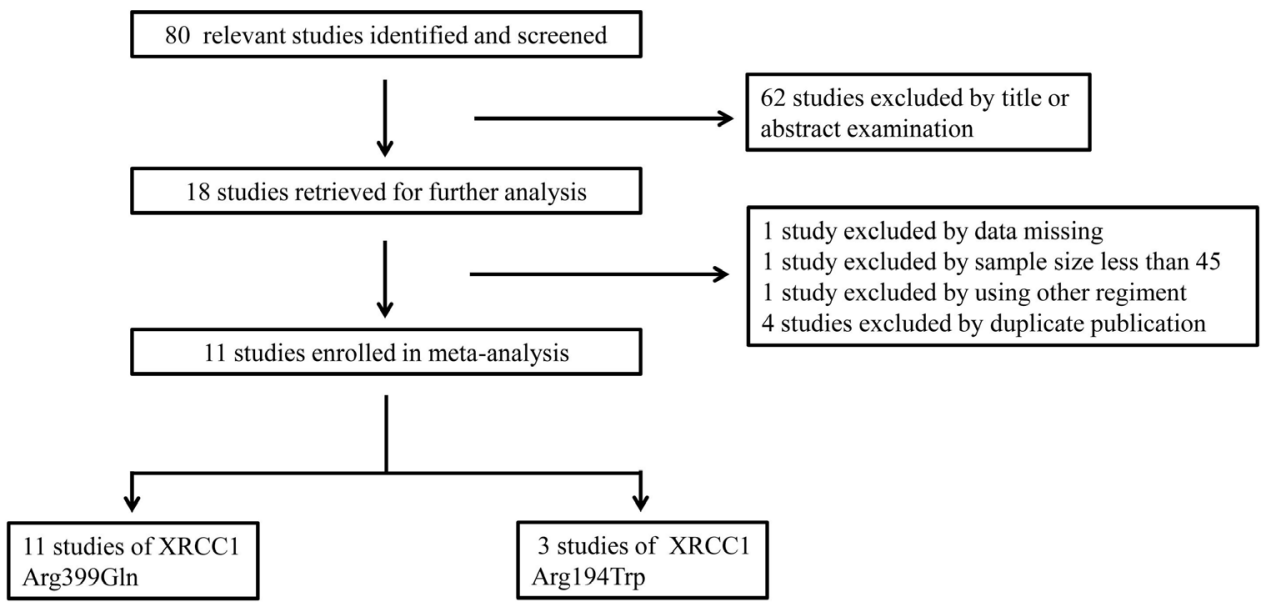

Figure 1. Flow chart of the study selection process for eligible articles.

\begin{tabular}{|c|c|c|c|c|c|c|c|c|c|}
\hline First author & Year & Country & Ethnicity & $\begin{array}{l}\text { Sample } \\
\text { sizes }\end{array}$ & $\begin{array}{c}\text { Median age } \\
\text { [years (min-max)] }\end{array}$ & $\begin{array}{c}\text { Evaluation } \\
\text { criterion }\end{array}$ & Methods & SNPs & Outcomes \\
\hline Ruzzo & 2006 & Italy & Caucasian & 175 & $61(38-79)$ & WHO & PCR-RFLP & $\begin{array}{l}\text { Arg399Gln } \\
\text { Arg194Trp }\end{array}$ & $\begin{array}{l}\text { TR } \\
\text { TR }\end{array}$ \\
\hline Keam & 2008 & Korea & Asian & 73 & $59(24-77)$ & WHO & PCR-PFLP & Arg399Gln & TR, PFS, OS \\
\hline Qiu & 2009 & China & Asian & 68 & $55(38-77)$ & WHO & PCR-RFLP & Arg399Gln & TR, PFS \\
\hline Huang & 2009 & China & Asian & 102 & $58(34-76)$ & - & PCR-RFLP & Arg399Gln & OS \\
\hline Goekkurt & 2009 & Germany & Caucasian & 193 & $64(27-86)$ & WHO & PCR-RFLP & Arg399Gln & TR, OS \\
\hline Shim & 2010 & Korea & Asian & 200 & $58(19-76)$ & RECIST & PCR-RFLP & $\begin{array}{l}\text { Arg399Gln } \\
\text { Arg194Trp }\end{array}$ & $\begin{array}{l}\text { TR, PFS, OS } \\
\text { TR }\end{array}$ \\
\hline Liang & 2010 & China & Asian & 81 & $55(32-77)$ & - & Taqman & Arg399Gln & PFS, OS \\
\hline $\mathrm{Ji}$ & 2010 & China & Asian & 59 & - & RECIST & PCR-RFLP & Arg399Gln & TR \\
\hline Gao & 2010 & China & Asian & 91 & $58(21-75)$ & WHO & PCR-RFLP & $\begin{array}{l}\text { Arg399Gln } \\
\text { Arg194Trp }\end{array}$ & $\begin{array}{l}\text { TR } \\
\text { TR }\end{array}$ \\
\hline Park & 2011 & Korea & Asian & 106 & $57(26-72)$ & RECIST & PCR-RFLP & Arg399Gln & PFS, OS \\
\hline Liu & 2011 & China & Asian & 126 & - & - & Taqman & Arg399Gln & OS \\
\hline
\end{tabular}

$\mathrm{TR}=$ therapeutic response; $\mathrm{PFS}=$ progression-free survival; $\mathrm{OS}=$ overall survival.

\section{Objective response}

There were seven eligible studies covering 809 individuals for the analysis of the predictive value of XRCC1 Arg399Gln concerning the objective response of gastric cancer to platinum-based treatment (Ruzzo et al., 2006; Keam et al., 2008; Goekkurt et al., 2009; Qiu and Wang, 2009; Gao et al., 2010; Ji et al., 2010; Shim et al., 2010). However, in the dominant model, the A allele did not show any positive association between polymorphism and treatment response in all patients $(\mathrm{G} / \mathrm{A}+\mathrm{A} / \mathrm{A} v s \mathrm{G} / \mathrm{G}: \mathrm{OR}=0.97 ; 95 \% \mathrm{CI}=0.49-1.26)($ Table 2$)$ or stratified analysis by ethnicity (Asian: $\mathrm{OR}=0.84 ; 95 \% \mathrm{CI}=0.42-1.70$; Caucasian: $\mathrm{OR}=0.75$; $95 \% \mathrm{CI}=0.38-1.48)$. No publication bias was observed, either by the Funnel test or the Egger test (data not shown). 
Table 2. Analysis of the association among XRCC1 Arg399Gln and Arg194Trp polymorphisms and objective response, progression-free survival (PFS), and overall survival (OS).

\begin{tabular}{|c|c|c|c|c|c|c|c|c|c|}
\hline & \multicolumn{3}{|c|}{ Objective response } & \multicolumn{3}{|c|}{ PFS } & \multicolumn{3}{|c|}{ OS } \\
\hline & Study (cases) & OR $(95 \% \mathrm{CI})$ & $\mathrm{P}_{\text {het }}^{\mathrm{b}}$ & Study (cases) & $\mathrm{HR}(95 \% \mathrm{CI})$ & $\mathrm{P}_{\text {het }} \mathrm{b}^{2}$ & Study (cases) & $\operatorname{HR}(95 \% \mathrm{CI})$ & $\mathrm{P}_{\text {het }}{ }^{\mathrm{b}}$ \\
\hline $\begin{array}{l}\mathrm{Arg} 399 \mathrm{Gln} \\
\mathrm{G} / \mathrm{A}+\mathrm{A} / \mathrm{A} v s \mathrm{G} / \mathrm{G} \\
\text { Arg194Trp }\end{array}$ & $7(809)$ & $0.79(0.49-1.26)^{\mathrm{c}}$ & 0.03 & $5(528)$ & $1.32(0.84-2.07)^{\mathrm{c}}$ & 0.00 & $7(823)$ & $1.40(1.04-1.90)^{\mathrm{c}}$ & 0.01 \\
\hline $\mathrm{C} / \mathrm{T}+\mathrm{T} / \mathrm{T}$ vs $\mathrm{C} / \mathrm{C}$ & $3(466)$ & $0.92(0.60-1.41)$ & 0.68 & - & - & - & - & - & - \\
\hline
\end{tabular}

Three eligible studies including 466 individuals were selected to confirm whether the XRCC1 Arg194Gln polymorphism was correlated with platinum-based chemotherapy response (Ruzzo et al., 2006; Gao et al., 2010; Shim et al., 2010). Unfortunately, there was no association between genetic variations and treatment response in any population $(\mathrm{C} / \mathrm{T}+\mathrm{T} / \mathrm{T}$ vs $\mathrm{C} / \mathrm{C}: \mathrm{OR}=0.92 ; 95 \% \mathrm{CI}=0.60-1.41)($ Table 2 ) or stratified analysis by ethnicity (Asian: OR $=0.94 ; 95 \% \mathrm{CI}=0.59-1.52$; Caucasian: $\mathrm{OR}=0.84 ; 95 \% \mathrm{CI}=0.33-2.12$ ). No publication bias was observed either by the Funnel test or the Egger test (data not shown).

\section{Progression-free survival}

Five studies concerning the predictive value of the XRCC1 Arg399Gln polymorphism with respect to PFS to the platinum-based treatment were selected for the final analysis (Keam et al., 2008; Qiu and Wang 2009; Liang et al., 2010; Shim et al., 2010; Park et al., 2011). Five hundred and twenty-eight gastric cancer patients enrolled in our studies were Asian. Unfortunately, the A allele did not have a higher PFS than the $\mathrm{G}$ allele when tested using the randomeffect model $(\mathrm{G} / \mathrm{A}+\mathrm{A} / \mathrm{A} v \mathrm{~s} / \mathrm{G}$ : $\mathrm{HR}=1.32 ; 95 \% \mathrm{CI}=0.84-2.07)$ (Table 2). No publication bias was observed either by the Funnel test or the Egger test (data not shown).

\section{Overall survival}

Seven studies covering 881 patients were eligible for the analysis of the association between the XRCC1 Arg399Gln polymorphism and OS (Keam et al., 2008; Goekkurt et al., 2009; Huang et al., 2009; Liang et al., 2010; Shim et al., 2010; Liu et al., 2011; Park et al., 2011). Only 1 study was based on the Caucasian population (Goekkurt et al., 2009). Patients with the A allele had a shorter overall survival time than those with the $\mathrm{G}$ allele when tested with the random-effect model $(\mathrm{G} / \mathrm{A}+\mathrm{A} / \mathrm{A}$ vs $\mathrm{G} / \mathrm{G}$ : $\mathrm{HR}=1.40 ; 95 \% \mathrm{CI}=1.04-1.90)$ (Figure 2 and Table 2). No publication bias was observed either by the Funnel test or the Egger test (Figure 3).

\section{DISCUSSION}

In this meta-analysis, we found that only gastric cancer patients with the XRCC1 399Gln allele, who were treated by platinum-based chemotherapy, had a negative association with overall survival time. 


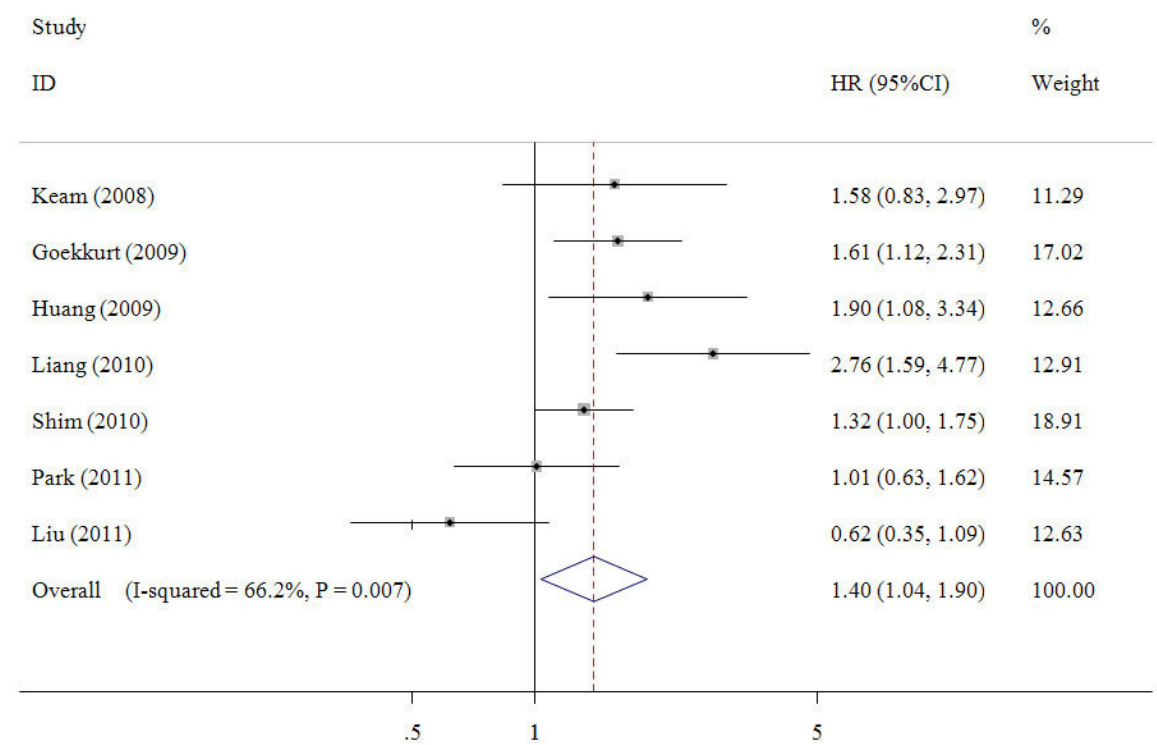

Figure 2. Forest plot of overall suvival in gastric cancer patients treated with platinum-based chemotherapy by the XRCC1 Arg399Gln polymorphism (G/A+A/A vs $\mathrm{G} / \mathrm{G}$, reference group $=\mathrm{G} / \mathrm{G})$.

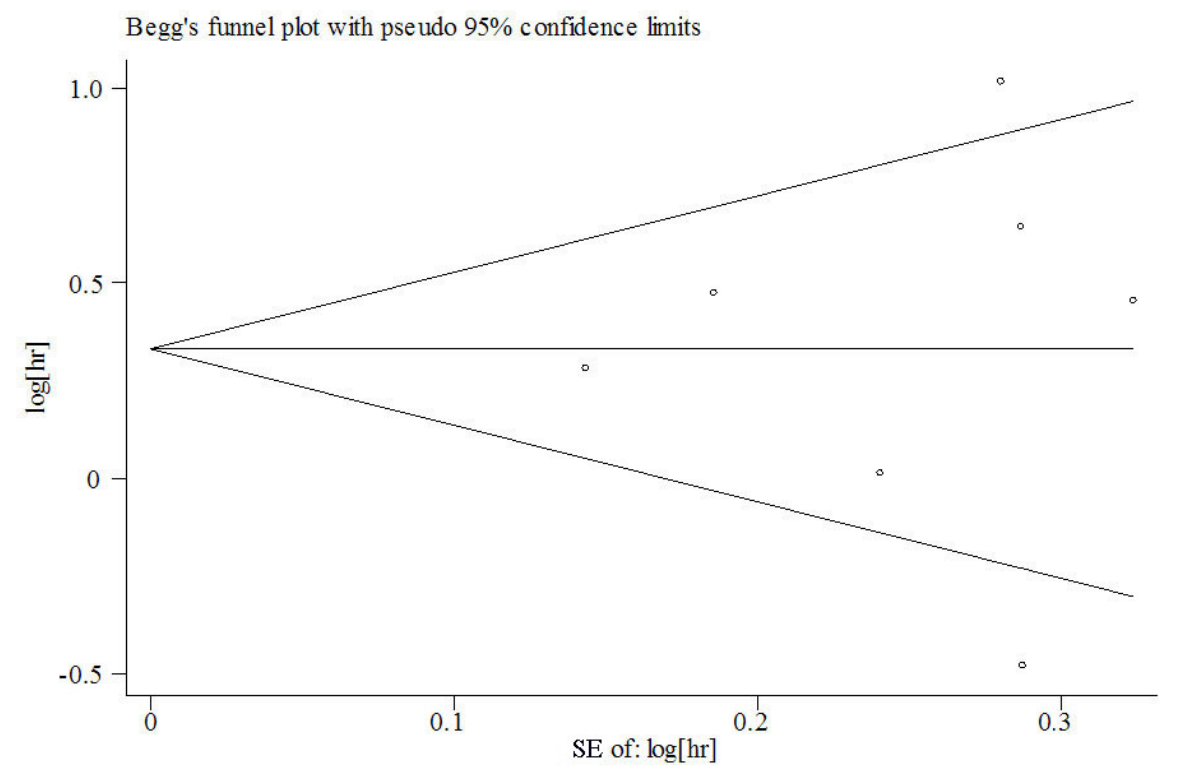

Figure 3. Funnel plot of overall suvival in gastric cancer patients treated with platinum-based chemotherapy by the XRCC1 Arg399Gln polymorphism (G/A+A/A vs $\mathrm{G} / \mathrm{G}$, reference group $=\mathrm{G} / \mathrm{G})$.

Patients with more resistance to platinum therapy have poorer clinical outcomes (d'Amato et al., 2009). Various mechanisms underlie platinum resistance, among which the DNA repair is one of the most predominant mechanisms (Rabik and Dolan, 2007; Galluzzi et al., 
2012; Shen et al., 2012). XRCC1 is an indispensable protein involved in DNA repair, playing a critical role in base-excision repair (Parsons et al., 2010), homologous recombination (Fan et al., 2007), and non-homologous end joining (Audebert et al., 2004). The XRCC1 Arg194Trp and Arg399Gln polymorphisms are the most studied SNPs and contribute to altering the DNA repair capacity, thus altering chemotherapy sensitivity and clinical outcome. Previous studies showed that XRCCl polymorphisms had predictive value of the clinical outcomes of lung cancer patients undergoing platinum-based chemotherapy (Cui et al., 2012; Wu et al., 2012). However, the predictive value for gastric cancer patients is still controversial, which prompted us to conduct the present meta-analysis to comprehensively evaluate the role of the XRCC1 polymorphisms.

In this meta-analysis, we assessed the $X R C C 1$ polymorphisms on the clinical outcomes of patients treated with platinum-based chemotherapy based on objective response, PFS, and OS. Because the objective response does not always correlate with survival outcome (Lokich, 2004), we analyzed objective response, PFS, and OS together for a comprehensive assessment. The XRCC1 Arg194Trp and Arg399Gln polymorphisms were not associated with the objective response, in the overall population or stratified by ethnicity. In addition, the XRCC1 Arg399Gln polymorphism did not show the predictive role in PFS. Interestingly, we found that patients with the XRCC1 Arg399 allele might have a shorter OS time when treated with platinum-based drugs, which was consistent with 4 of the included studies (Goekkurt et al., 2009; Huang et al., 2009; Liang et al., 2010; Shim et al., 2010). Because only 1 in 7 studies was conducted in Caucasians, additional studies with larger sample sizes and different ethnic backgrounds are needed to make a more definitive conclusion.

Above all, we should clarify that there were several limitations in this meta-analysis, despite that Egger and Begg tests suggested that potential publication bias may not exist. First, only 2 studies were based on the Caucasian population (Table 1), which might cause bias. Moreover, there were no associations between the XRCC1 Arg194Trp and Arg399Gln polymorphisms, objective response, and PFS in Caucasian patients. Although we repeatedly contacted the corresponding author for their original data, we could not get the data for final analysis of PFS (Ruzzo et al., 2006; Goekkurt et al., 2009; Ott et al., 2011). The conclusion was unlikely to change, as the results of the PFS outcome in the 3 studies with missing data were consistent with ours. However, the OS outcome conclusion might be altered marginally. Secondly, different chemotherapy regimens combined with platinum may affect the outcomes. However, we were not able to estimate the impact on the conclusions when different chemotherapy drugs were applied in the recruited data. Thirdly, due to the differences in study design, we could not perform detailed stratification analysis.

In conclusion, our meta-analysis indicated that the XRCC1 Arg399Gln polymorphism may be a prognostic biomarker for OS for platinum-based gastric cancer treatment. However, well-designed studies with larger sample sizes using individuals with different genetic backgrounds are needed to confirm these findings.

\section{ACKNOWLEDGMENTS}

Research supported by the National Natural Science Foundation of China (\#30930080, \#81161120537, and \#81001231), the Natural Science Foundation of Jiangsu Province (\#BK2012840), the Postdoctoral Science Foundation of China (\#20100481165), Under- 
graduates Practice and Innovation Training Project of Jiangsu Higher Education Institutes (\#2012JSSPITP1021), and the Priority Academic Program Development of Jiangsu Higher Education Institutions (Public Health and Preventive Medicine).

\section{Conflicts of interest}

The authors declare no conflict of interest.

\section{REFERENCES}

Audebert M, Salles B and Calsou P (2004). Involvement of poly(ADP-ribose) polymerase-1 and XRCC1/DNA ligase III in an alternative route for DNA double-strand breaks rejoining. J. Biol. Chem. 279: 55117-55126.

Cui Z, Yin Z, Li X, Wu W, et al. (2012). Association between polymorphisms in XRCC1 gene and clinical outcomes of patients with lung cancer: a meta-analysis. BMC Cancer 12: 71.

d'Amato TA, Pettiford BL, Schuchert MJ, Parker R, et al. (2009). Survival among patients with platinum resistant, locally advanced non-small cell lung cancer treated with platinum-based systemic therapy. Ann. Surg. Oncol. 16: 2848-2855.

David SS, O'Shea VL and Kundu S (2007). Base-excision repair of oxidative DNA damage. Nature 447: 941-950.

Deans AJ and West SC (2011). DNA interstrand crosslink repair and cancer. Nat. Rev. Cancer 11: 467-480.

Fan J, Wilson PF, Wong HK, Urbin SS, et al. (2007). XRCC1 down-regulation in human cells leads to DNA-damaging agent hypersensitivity, elevated sister chromatid exchange, and reduced survival of BRCA2 mutant cells. Environ. Mol. Mutagen. 48: 491-500.

Galluzzi L, Senovilla L, Vitale I, Michels J, et al. (2012). Molecular mechanisms of cisplatin resistance. Oncogene 31: 1869-1883.

Gao C, Chen H, Lu J and Ding J (2010). Relationship between polymorphisms of XRCC1 gene and sensitivity to chemotherapy in advanced gastric cancer. Chin. J. Clin. Ration. Drug Use 3: 4-6.

Goekkurt E, Al-Batran SE, Hartmann JT, Mogck U, et al. (2009). Pharmacogenetic analyses of a phase III trial in metastatic gastroesophageal adenocarcinoma with fluorouracil and leucovorin plus either oxaliplatin or cisplatin: a study of the arbeitsgemeinschaft internistische onkologie. J. Clin. Oncol. 27: 2863-2873.

Huang ZH, Hua D and Du X (2009). Polymorphisms in p53, GSTP1 and XRCC1 predict relapse and survival of gastric cancer patients treated with oxaliplatin-based adjuvant chemotherapy. Cancer Chemother. Pharmacol. 64: 10011007.

Ji M, Wu CP, Jiang JT and Xu B (2010). Relationship between polymorphism of X-ray repair cross-complementing group 1 (XRCC1) genes and clinical outcome of advanced gastric cancer patients treated with DDP-based chemotherapy. Chin. J. Clin. Oncol. 37: 945-947.

Keam B, Im SA, Han SW, Ham HS, et al. (2008). Modified FOLFOX-6 chemotherapy in advanced gastric cancer: Results of phase II study and comprehensive analysis of polymorphisms as a predictive and prognostic marker. BMC Cancer 8: 148.

Lau J, Ioannidis JP and Schmid CH (1997). Quantitative synthesis in systematic reviews. Ann. Intern. Med. 127: 820-826.

Liang J, Li QF, Yao RY, Lu HY, et al. (2010). Association between genetic polymorphisms of ERCC1, XRCC1, GSTP1 and survival of advanced gastric cancer patients treated with oxaliplatin/5-Fu-based chemotherapy. Zhonghua Zhong. Liu Za Zhi. 32: 515-519.

Liu YP, Ling Y, Zhang YP and Liu BR (2011). Predictive values of platinum-related gene polymorphisms in gastric cancer patients on oxaliplatin-based adjuvant chemotherapy. Zhonghua Yi. Xue Za Zhi. 91: 256-259.

Lokich J (2004). Tumor response and survival end points in clinical trials: a clinician's perspective. Am. J. Clin. Oncol. 27: 494-496.

Miller AB, Hoogstraten B, Staquet M and Winkler A (1981). Reporting results of cancer treatment. Cancer 47: 207-214.

Ott K, Rachakonda PS, Panzram B, Keller G, et al. (2011). DNA repair gene and MTHFR gene polymorphisms as prognostic markers in locally advanced adenocarcinoma of the esophagus or stomach treated with cisplatin and 5-fluorouracil-based neoadjuvant chemotherapy. Ann. Surg. Oncol. 18: 2688-2698.

Paoletti X, Oba K, Burzykowski T, Michiels S, et al. (2010). Benefit of adjuvant chemotherapy for resectable gastric cancer: a meta-analysis. JAMA 303: 1729-1737.

Park SR, Kong SY, Nam BH, Choi IJ, et al. (2011). CYP2A6 and ERCC1 polymorphisms correlate with efficacy of S-1 plus cisplatin in metastatic gastric cancer patients. Br. J. Cancer 104: 1126-1134. 
Parkin DM, Bray F, Ferlay J and Pisani P (2005). Global cancer statistics, 2002. CA Cancer J. Clin. 55: 74-108.

Parmar MK, Torri V and Stewart L (1998). Extracting summary statistics to perform meta-analyses of the published literature for survival endpoints. Stat. Med. 17: 2815-2834.

Parsons JL, Dianova II, Finch D, Tait PS, et al. (2010). XRCC1 phosphorylation by CK2 is required for its stability and efficient DNA repair. DNA Repair 9: 835-841.

Qiu D and Wang F (2009). Polymorphism of XRCC1 gene influences response to oxaliplatin-based chemotherapy in patients with advanced gastric cancer. J. Radioimmunol. 22: 630-632.

Rabik CA and Dolan ME (2007). Molecular mechanisms of resistance and toxicity associated with platinating agents. Cancer Treat. Rev. 33: 9-23.

Ruzzo A, Graziano F, Kawakami K, Watanabe G, et al. (2006). Pharmacogenetic profiling and clinical outcome of patients with advanced gastric cancer treated with palliative chemotherapy. J. Clin. Oncol. 24: 1883-1891.

Sakuramoto S, Sasako M, Yamaguchi T, Kinoshita T, et al. (2007). Adjuvant chemotherapy for gastric cancer with S-1, an oral fluoropyrimidine. N. Engl. J. Med. 357: 1810-1820.

Shen DW, Pouliot LM, Hall MD and Gottesman MM (2012). Cisplatin resistance: a cellular self-defense mechanism resulting from multiple epigenetic and genetic changes. Pharmacol. Rev. 64: 706-721.

Shim HJ, Yun JY, Hwang JE, Bae WK, et al. (2010). BRCA1 and XRCC1 polymorphisms associated with survival in advanced gastric cancer treated with taxane and cisplatin. Cancer Sci. 101: 1247-1254.

Therasse P, Arbuck SG, Eisenhauer EA, Wanders J, et al. (2000). New guidelines to evaluate the response to treatment in solid tumors. European Organization for Research and Treatment of Cancer, National Cancer Institute of the United States, National Cancer Institute of Canada. J. Natl. Cancer Inst. 92: 205-216.

Tierney JF, Stewart LA, Ghersi D, Burdett S, et al. (2007). Practical methods for incorporating summary time-to-event data into meta-analysis. Trials 8: 16.

Wang S, Wu X, Chen Y, Zhang J, et al. (2012). Prognostic and predictive role of JWA and XRCC1 expressions in gastric cancer. Clin. Cancer Res. 18: 2987-2996.

Wilting RH and Dannenberg JH (2012). Epigenetic mechanisms in tumorigenesis, tumor cell heterogeneity and drug resistance. Drug Resist. Updat. 15: 21-38.

Wu J, Liu J, Zhou Y, Ying J, et al. (2012). Predictive value of XRCC1 gene polymorphisms on platinum-based chemotherapy in advanced non-small cell lung cancer patients: a systematic review and meta-analysis. Clin. Cancer Res. 18: 3972-3981.

Zhu G and Lippard SJ (2009). Photoaffinity labeling reveals nuclear proteins that uniquely recognize cisplatin-DNA interstrand cross-links. Biochemistry 48: 4916-4925. 